\title{
Motion Cueing Algorithm Modification for Improved Turbulence Simulation
}

\author{
Anthony V. Ercole ${ }^{1}$, Frank M. Cardullo ${ }^{2}$, Kirill Zaychik ${ }^{3}$ \\ Man-Machine Systems Laboratory, Department of Mechanical Engineering, SUNY Binghamton, Binghamton, NY, \\ 13902-6000 \\ Lon Kelly ${ }^{4}$ \\ Unisys Corporation, Hampton, VA, 23666 \\ and \\ Jacob Houck ${ }^{5}$ \\ NASA LaRC, Hampton, VA, 23665
}

\begin{abstract}
Atmospheric turbulence cueing produced by flight simulator motion systems has been less than satisfactory because the turbulence profiles have been attenuated by the motion cueing algorithms. Cardullo and Ellor initially addressed this problem by directly porting the turbulence model output to the motion system. Reid and Robinson addressed the problem by employing a parallel aircraft model, which is only stimulated by the turbulence inputs and adding a filter specially designed to pass the higher turbulence frequencies.

There have been advances in motion cueing algorithm development at the Man-Machine Systems Laboratory, at SUNY Binghamton. In particular, the system used to generate turbulence cues has been studied. The Reid approach, implemented by Telban and Cardullo, was employed to augment the optimal motion cueing algorithm installed at the NASA LaRC Simulation Laboratory, driving the Visual Motion Simulator.

In this implementation, the output of the primary flight channel was added to the output of the turbulence channel and then sent through a non-linear cueing filter. The cueing filter is an adaptive filter; therefore, it is not desirable for the output of the turbulence channel to be augmented by this type of filter. The likelihood of the signal becoming divergent was also an issue in this design.

After testing on-site it became apparent that the architecture of the turbulence algorithm was generating unacceptable cues. As mentioned above, this cueing algorithm comprised a filter that was designed to operate at low bandwidth. Therefore, the turbulence was also filtered, augmenting the cues generated by the model. If any filtering is to be done to the turbulence, it will utilize a filter with a much higher bandwidth, above the frequencies produced by the aircraft response to turbulence.

The authors have developed an implementation wherein only the signal from the primary flight channel passes through the nonlinear cueing filter. This paper discusses three new algorithms. Testing shows that the new methods provide the pilot with a more realistic sensation of turbulence; the cues are not attenuated by algorithm. Results of offline testing show the credibility of the models. Offline test verification was based primarily on the evaluation of the power spectral density of the outputs and the time response.
\end{abstract}

${ }^{1}$ Graduate Student, Department of Mechanical Engineering, Student Member

${ }^{2}$ Professor, Department of Mechanical Engineering, Associate Fellow AIAA

${ }^{3} \mathrm{PhD}$ Candidate, Department of Mechanical Engineering, Student member

${ }^{4}$ Software Engineer, Simulation and Development Branch.

${ }^{5}$ Distinguished Research Associate, Simulation Development and Analysis Branch, Associate Fellow

1

American Institute of Aeronautics and Astronautics 


\section{Introduction}

This paper discusses a means to provide more realistic motion cues to the pilot of a flight simulator when the simulated aircraft is subjected to atmospheric turbulence or any other disturbance at frequencies that are above those of pilot control dynamics. A statement of the problem is presented along with a history of previous attempts at resolving this cueing ambiguity.

After a discussion of the background to the problem three potential solutions are discussed. These three implementations all include a separate cueing channel for turbulence in parallel with the primary cueing channel. The primary cueing channel is the channel that primarily responds to pilot control input and employs in the case a nonlinear adaptive drive algorithm. The first implementation does not include a washout filter in the turbulence channel while the second implementation does. The third implementation does not employ an aircraft model in the turbulence channel but instead attempts to extract the turbulence response from the combined aircraft response.

A brief discussion is included illustrating the design of a reduced order aircraft model which may be employed in the turbulence channel to reduce the real-time computational burden. The results section presents an analytical comparison of the first two implementations in both the time and frequency domains. The latter is effected by a plot of the power spectral density as a function of frequency.

The paper concludes with a summary of the results and a discussion of piloted testing of the two approaches.

\section{Background}

Historically there has been a problem with providing turbulence cues via platform motion systems due to the presence of motion cueing washout filters designed to washout the cue arising from pilot control. Iteration rate smoothing filters also present a problem in this regard. In the mid seventies one of us (FMC) developed an approach whereby the turbulence state bypassed the cueing algorithm and the iteration rate filter. In the early nineties Prof. Lloyd Ried and his students at the University of Toronto developed a different approach (Reid and Robinson). As

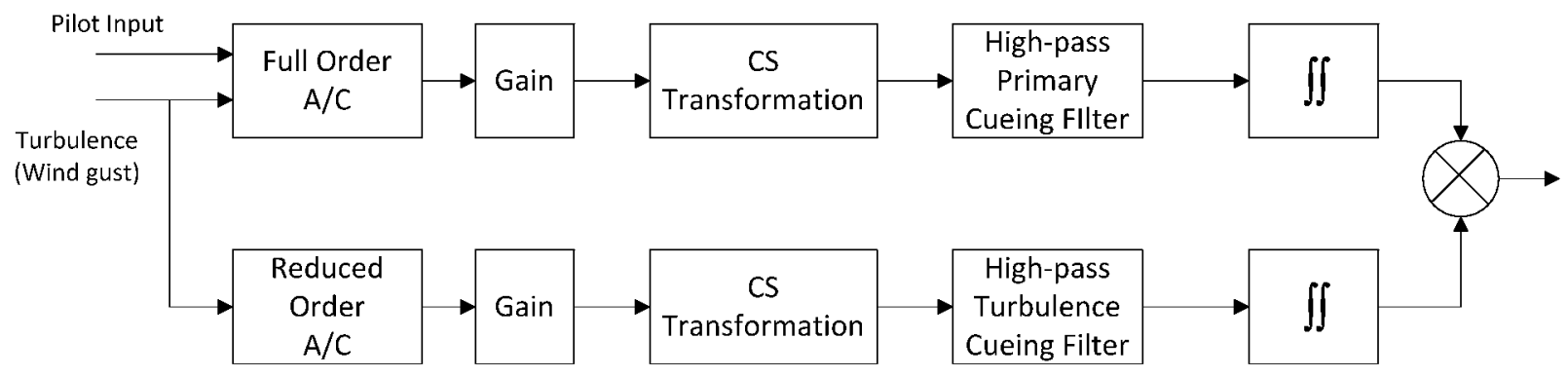

Figure 1 University of Toronto augmented motion cueing algorithm (Reid and Robinson)

can be seen, their approach introduced a second channel which employed a reduced order model of the aircraft's response to turbulence. The aircraft response to turbulence was then passed through a high-pass filter, different from the primary cueing high-pass filter. The filter output was then integrated and summed with the primary cueing output and sent to the motion system hardware. A unique attribute of the Toronto approach is to use a reduced order model of the aircraft so as to render the aircraft response to the turbulence input without the burden of the computation required with a second full order aircraft model. 
During the development of a non-linear cueing algorithm in our laboratory we sought to incorporate the University of Toronto approach. . Figure 2 illustrates the approach taken, which was in fact an incorrect implementation of what was intended. It can be seen in this implementation that the turbulence response to the aircraft was subjected to the same cueing filter as the pilot control albeit with a different gain. The assumption was that since the filter was nonlinear and adaptive with the parameters adjusted in real-time based on perceptual error,

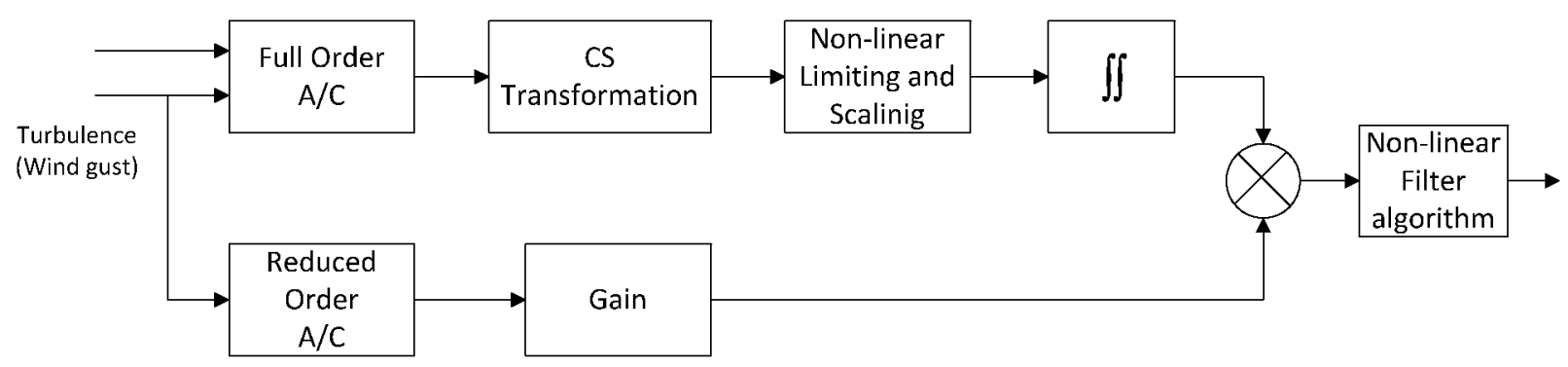

Figure 2 SUNY Binghamton initial implementation (Telban \& Cardullo)

this would be equivalent to the Reid approach. However the fallacy in that assumption is twofold; (a) the vestibular system model used in the cueing algorithm did not include response to frequencies in the range of aircraft response to turbulence. (b) The aircraft response to turbulence is not necessarily a zero mean process and therefore the double integration might cause the amplitude of the response of the motion platform to grow with time.

The Simulation Development and Analysis Branch at NASA Langley Research Center (NASA LaRC) employs two atmospheric turbulence models. The one used in verifying this simulation is the Full Dryden Model. This model was generated from data that was obtained when flying an aircraft through atmospheric turbulence. This particular simulation can also run using the Simple Dryden Model. The Simple Dryden utilizes a pseudo random number generator to produce turbulent wind gusts within the spectrum of atmospheric turbulence. Like the Full Dryden, the level of intensity can be varied from 1 to 5(check if it is 6).

The reason for using the Full Dryden model was to ensure repeatability. Since the data are prerecorded, it is very easy to vary the turbulence level and repeat tests using the same gust input.

\section{New Algorithm Methodology}

\section{Proposed Implementations}

Two new algorithms are proposed, each of which contains a primary and secondary flight channel. Both flight channels have a full order, nonlinear, aircraft model (FOAC). The primary channel takes pilot control input along with atmospheric turbulence as inputs. The secondary flight channel is designed to produce the aircraft's reaction to atmospheric turbulence in the vertical direction; therefore, it only receives turbulence wind gust data as an input.

In the first implementation, shown in Figure 3, the secondary flight channel comprises both a FOAC along with a specially designed cueing filter. Implementation 2 is identical to this except for the removal of the cueing filter, see Figure 4. If determined to be unnecessary, the filter can be removed from the turbulence channel.

The output of the secondary flight channel is not summed with the primary flight channel until after it passes through the motion cueing algorithm. This includes the CS Transformation, Nonlinear Limiting and Scaling, Nonlinear Filter Algorithm, and integrations. 


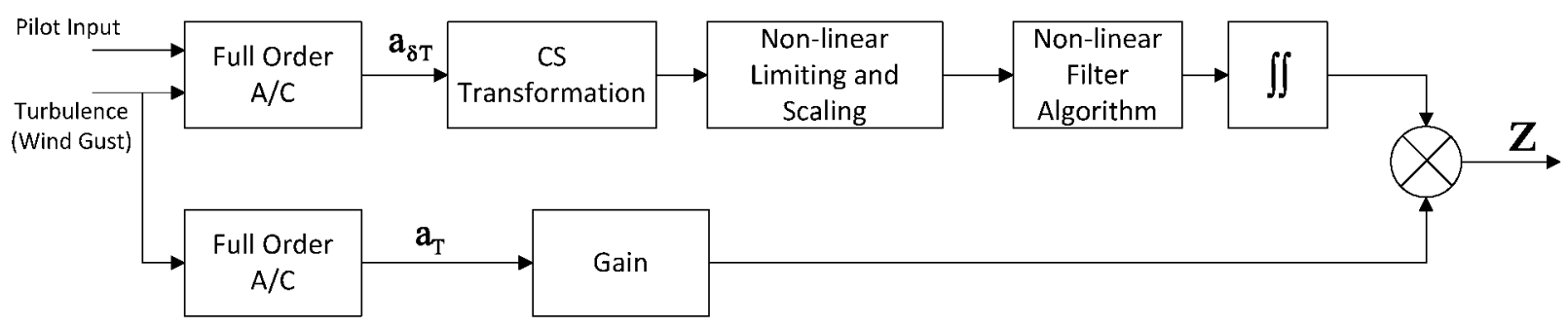

Figure 3: Proposed Implementation 1

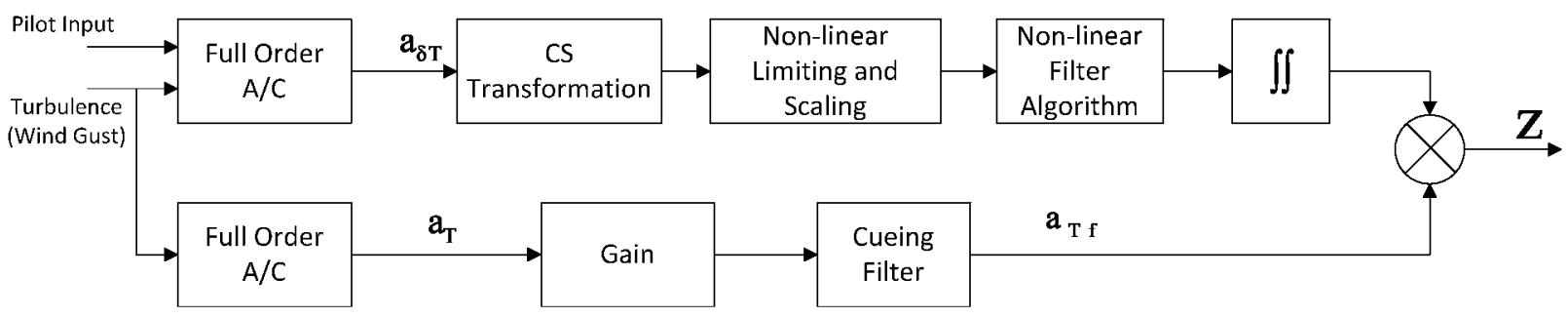

Figure 4: Proposed Implementation 2

Alternate representations of both implementation 1 and 2 are shown in figures 5 and 6 . If the computational burden is too great when using two FOAC models, the model in the turbulence channel can be replaced with a linear, Reduced Order Aircraft Model (ROAC, as in the original Binghamton implementation (Telban \& Cardullo).

The FOAC represents the dynamics of a Boeing 757. The model was provided by NASA Langley Research Center. The full order dynamics were used to obtain a basis of verification and development of the ROAC. This robust interactive model can be set to record various outputs and trimmed to almost any flight conditions. Specifically, these runs were trimmed to initial conditions representing landing approach. The same conditions were used in experiments at NASA.

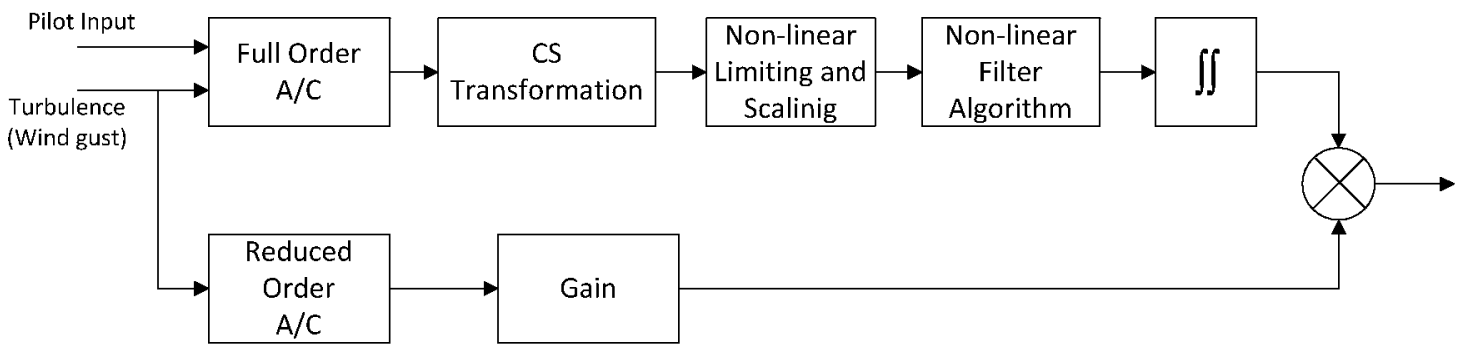

Figure 5: Implementation 1 with ROAC

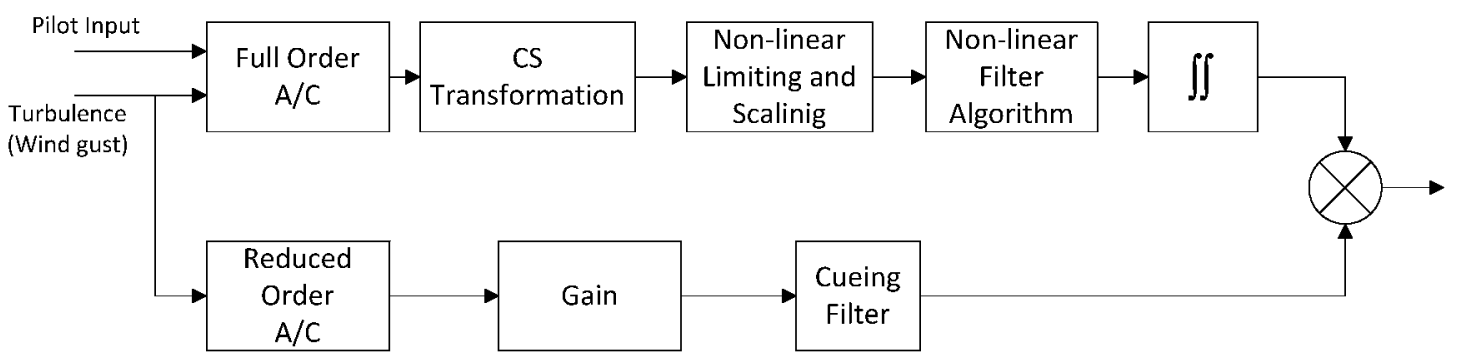

Figure 6: Implementation 2 with ROAC 
A third possible implementation, with no aircraft model in the turbulence channel has also been proposed in some quarters. In this approach, the secondary flight channel contains a Turbulence Filtering Algorithm, used to obtain turbulence cues directly from the full order aircraft model. Since the FOAC generates the aircraft response to turbulence and pilot control, it is thought that the specific aircraft response to turbulence can be obtained from the output by filtering the pilot control induced dynamics in the secondary flight channel, see figure 7 . The problem with this is that the frequencies induced by pilot control and turbulence overlap considerably.

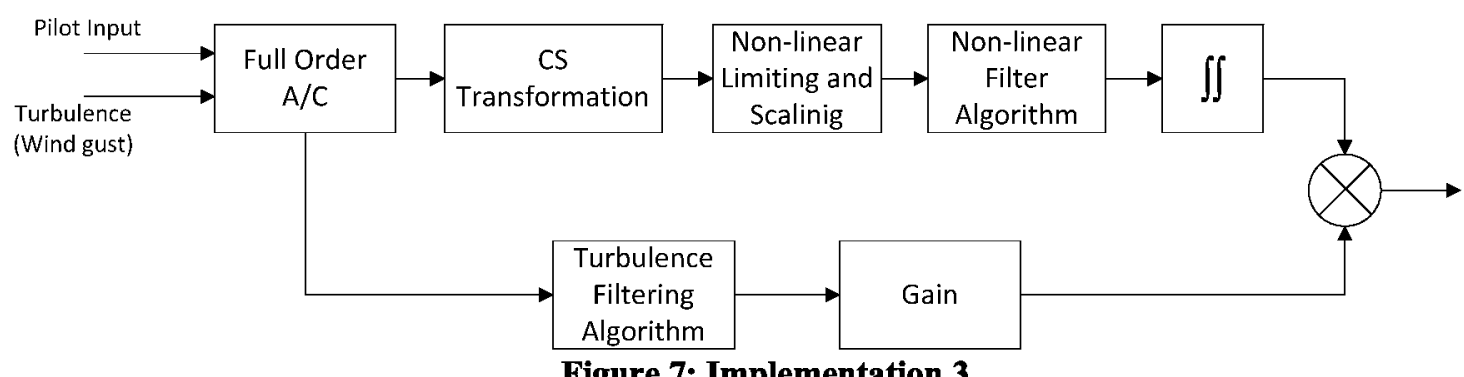

Figure 7: Implementation 3

\section{Cueing Filter Design}

The original cueing filter was not designed to attenuate the high frequency input signals. It also generated considerable phase change in the output signal. Applying the original filter to the turbulence channel would result in considerable phase change along with added gain. This led to a redesign of the filter.

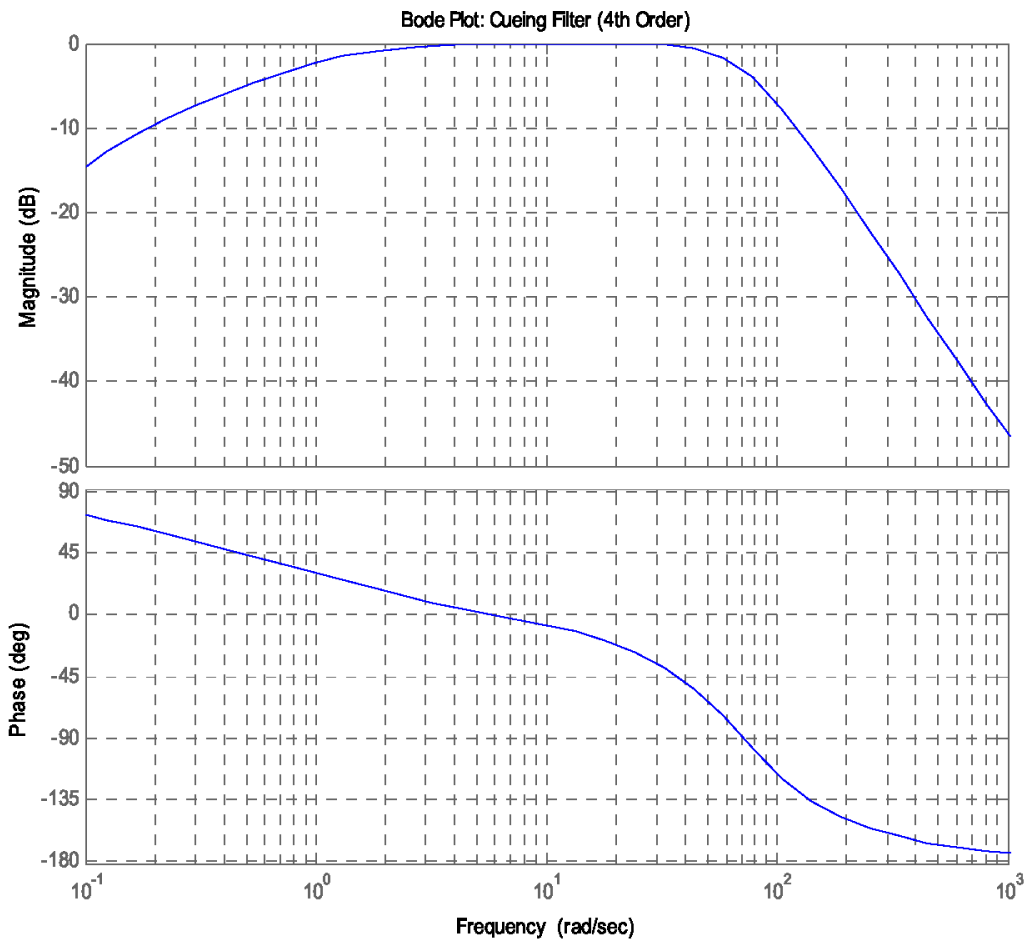

The newly designed cueing filter serves many purposes; see Equation 1 for transfer function. It has a very fast rise time, making it extremely responsive to any high frequency input. The step response settles at zero, effectively washing out any signal accumulation. With a settling time of approximately 10 seconds, see figure 8, low frequency oscillation of the aircraft's vertical acceleration is removed from the signal, see Figure xx. The long period dynamics of the aircraft lead to this acceleration.

No gain is added to the signal for the main spectrum of turbulence frequencies. From about $1 \mathrm{rad} / \mathrm{s}$ to $60 \mathrm{rad} / \mathrm{s}$, the gain remains at $0 \mathrm{~dB}$, providing no modification to the input signal, see Figure xx. The signal will be attenuated at greater frequencies, adding to the stability of the system, see figure 9 . 


$$
\frac{\mathrm{a}_{\mathrm{T}}(s)}{\mathrm{a}_{\mathrm{Tf}}(s)}=\frac{\begin{array}{c}
\text { Transfer function of new cueing filter } \\
0.0002041 \\
s^{4}+0.02026 s^{3}+1.025 s^{2}+1.255 s+0.25
\end{array}}{}
$$

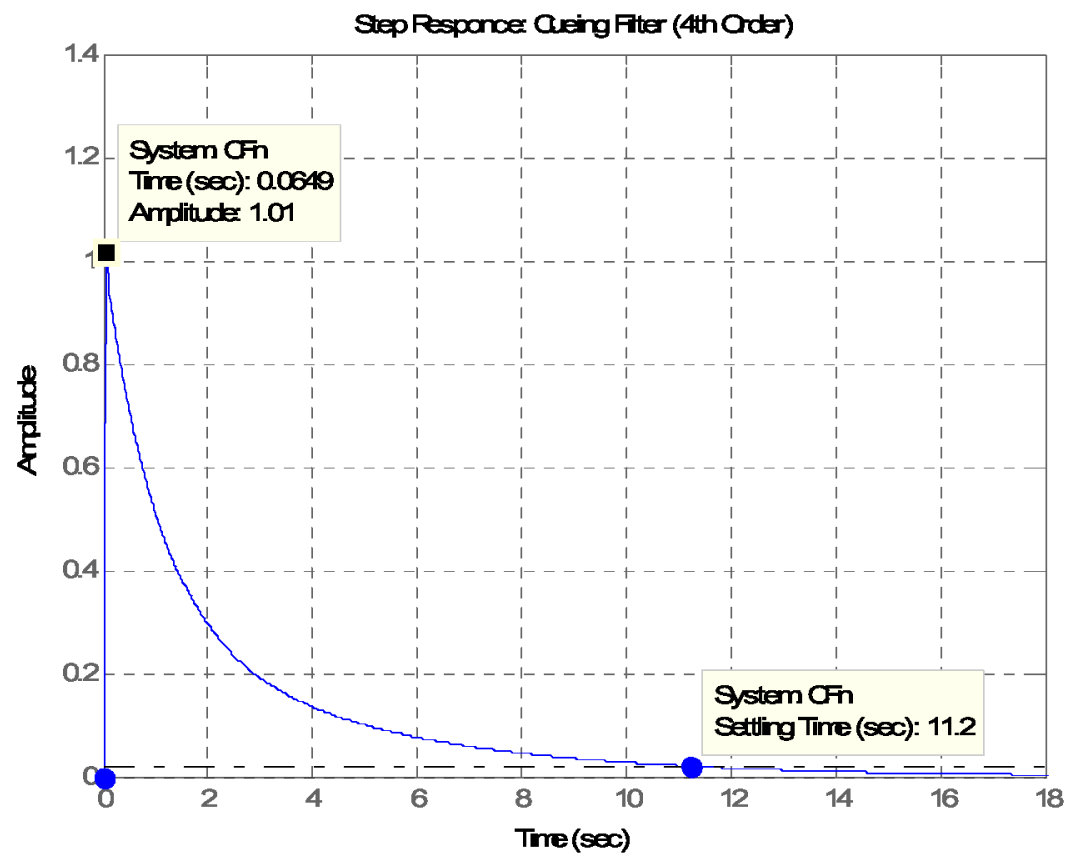

Figure 8: Step Response of the Cueing Filter, showing the Rise

\section{Reduced Order Aircraft Model Design}

In two implementations a ROAC is used in the turbulence channel as opposed to a full order model. This is done for many reasons, including reduced computation time. Furthermore, since the secondary flight channel only comprises one channel of motion, a linear model can sufficiently obtain the aircraft's acceleration.

To generate the reduced order aircraft model two methods were used. The first being the AutoRegressive with eXternal input (ARX) model estimator. This is a highly robust estimator that uses least-squares analysis to estimate the parameters of the model. The identified model represents the aircraft acceleration in the vertical direction, in response to a vertical turbulent gust input. There was not much success preserving the dynamics of the full order aircraft model using this estimator. The time response was somewhat accurate but the frequency of the signal was not maintained. See figure $\mathrm{xx}$ for the transfer function of the model.

The second approach was to use a method developed at the Man-Machine Systems Laboratory at Binghamton University. Automatic Parameter Identification (APID) is an algorithm that identifies a model based on the Power Spectral Density (PSD) of supplied signals. Preserving the PSD of the aircraft reaction to turbulence is a high priority in this simulation. The turbulence cues are sent to the pilot in the form of acceleration. Preserving the 
frequency and amplitude of the acceleration ensures proper cues will be sent to the motion system. Multiple models were generated in an attempt to determine the most refined model, shown in equations 2 and 3.

\section{ARX ROAC Transfer Function}

$$
\mathrm{a}_{\mathrm{T}}(s)=\frac{.2422 s^{3}+34 s^{2}-845.5 s+2829}{s^{4}+126.7 s^{3}+9762 s^{2}+3.855 * 10^{5} s+8,964 * 10^{4}} \text {, }
$$

$$
\begin{gathered}
\text { APID ROAC Transfer Function } \\
\mathrm{a}_{\mathrm{T}}(s)=\frac{819.1 s^{3}+358.8 s^{2}+204.7 s+403.1}{s^{4}+9830 s^{3}+2.616 * 10^{4} s^{2}+2.13 * 10^{4} s+3439},
\end{gathered}
$$

This section contains the results of various verification tests conducted on each of the new implementations.

\section{Power Spectral Density of ROAC}

The PSD of each ROAC is shown in Figures 10 and 11. It is obvious that the model produced using APID follows the PSD of the FOAC much better than the ARX model. The time response of this ROAC is only satisfactory for implementation 2, with the cueing filter in the turbulence channel. The washout effect, brought upon by the cueing filter allows both the time response and PSD of the ROAC and FOAC to become almost indistinguishable.

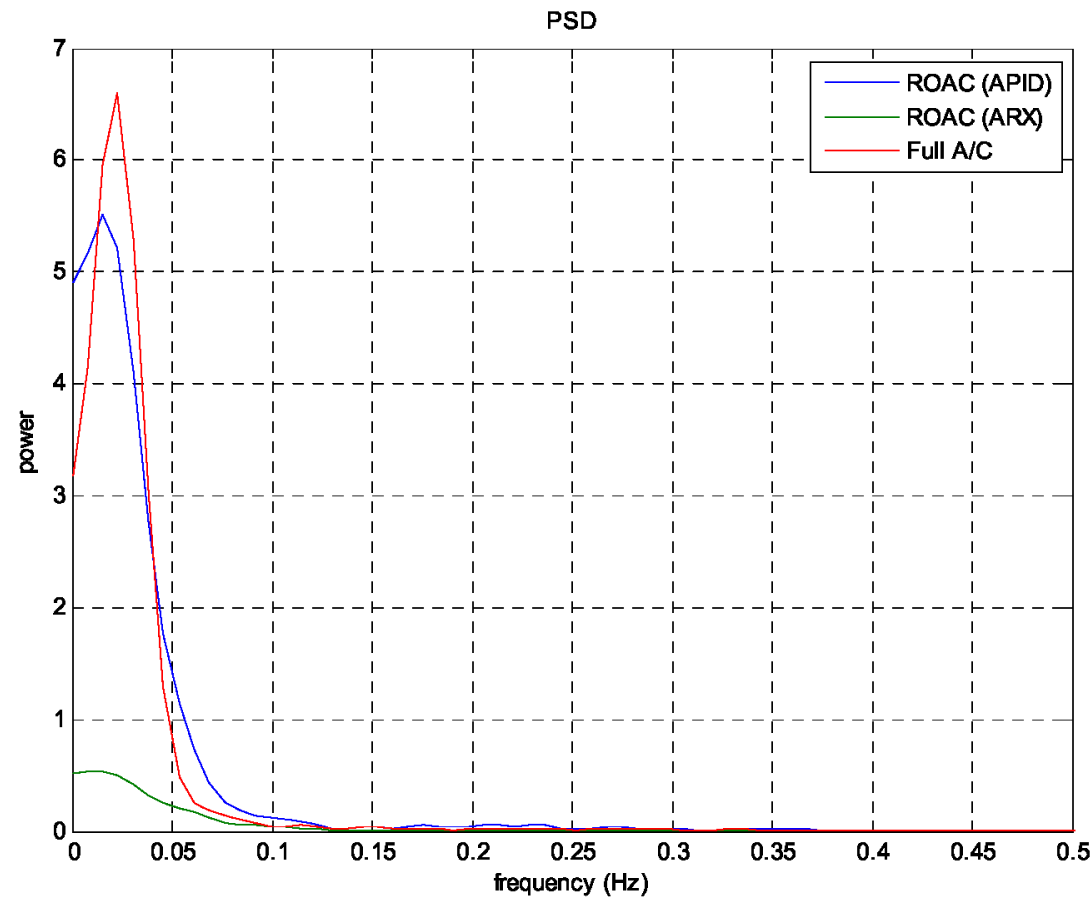

Figure 10: PSD of the ROAC generated using APID, the ROAC generated using ARX, and the FOAC 


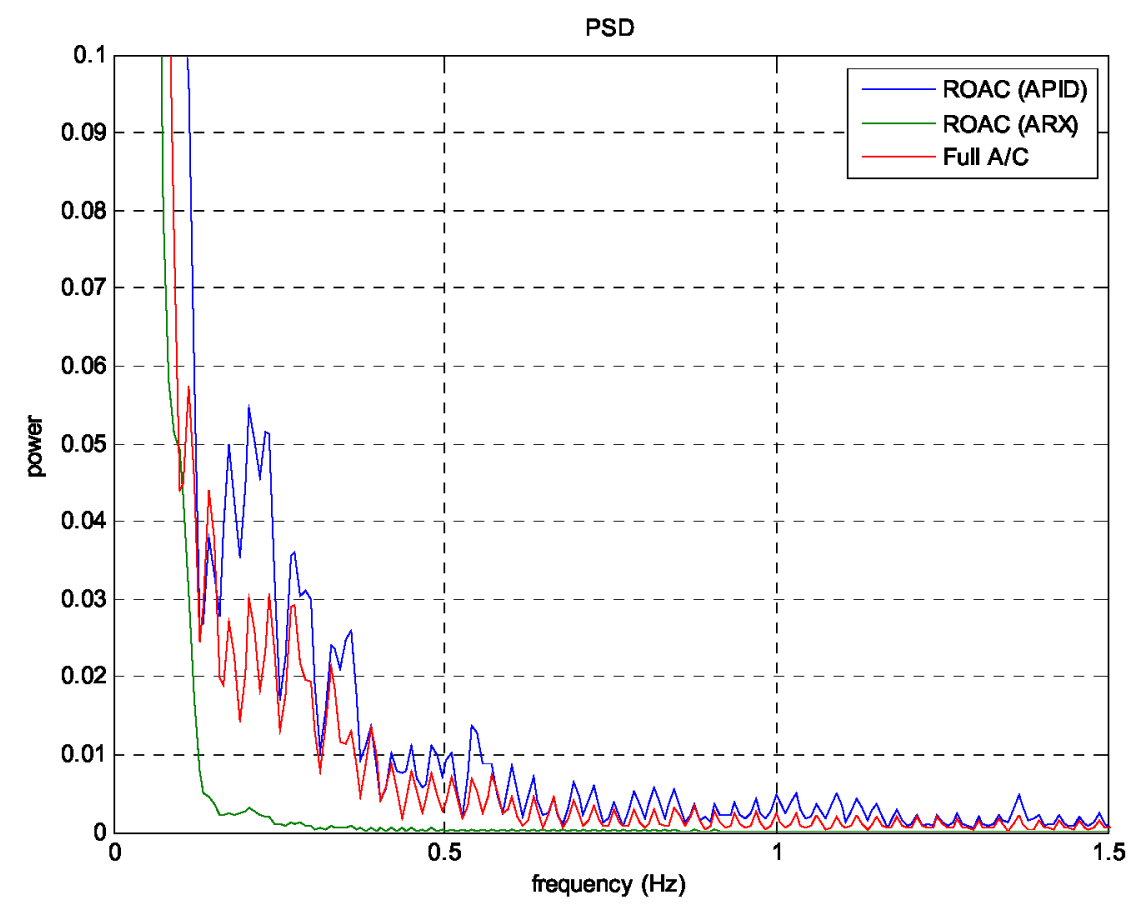

Figure 11: Zoomed view of the PSD of the ROAC generated using APID, the ROAC generated using ARX, and the FOAC

\section{Results}

\section{Analytical Results of Implementation 1 and 2}

The simulation was modified to ensure the motion cueing algorithm generated proper acceleration cues. To verify the simulation, the motion cue generated by the algorithm $(\mathrm{Z})$ is compared to the data produced by the full order aircraft model. The motion cueing algorithm portion of the implementation includes the CS Transformation, Non-linear Limiting and Scaling, Non-linear Filtering Algorithm, and two integrations. It is here where the turbulence cues are augmented, which is what constitutes the need for the turbulence channel.

Referring to Figure 12, shown here is the time response of three key calculations taking place within the simulation. First, the FOAC generates the vertical acceleration of the aircraft due to both turbulence and pilot input $\left(a_{\delta T}\right)$. For this case, the pilot input is a sinusoidal pitch stick input, covering one cycle, with a period of two seconds and an amplitude of $15 \%$ of the maximum pitch stick. ).

The second calculation is the vertical acceleration of the aircraft due to turbulence alone $\left(a_{T}\right)$, and finally, the output of the entire simulation $(Z)$. Since $Z$ is a position cue, sent to the motion system, it is measured in meters. $a_{\delta T}$ $\mathrm{a}_{\mathrm{T}}$ are aircraft accelerations, and have the units, $\mathrm{m} / \mathrm{s}^{2}$.

$\mathrm{Z}$ represents the cue sent directly to the motion system of the simulator. For the cues to be perceived correctly, there must be solid representation of both atmospheric turbulence and pilot input. This legitimizes the comparison of $\mathrm{Z}$ to $\mathrm{a}_{\delta \mathrm{T}}$, being that $\mathrm{a}_{\delta \mathrm{T}}$ contains the full dynamics of the aircraft with pilot input. 


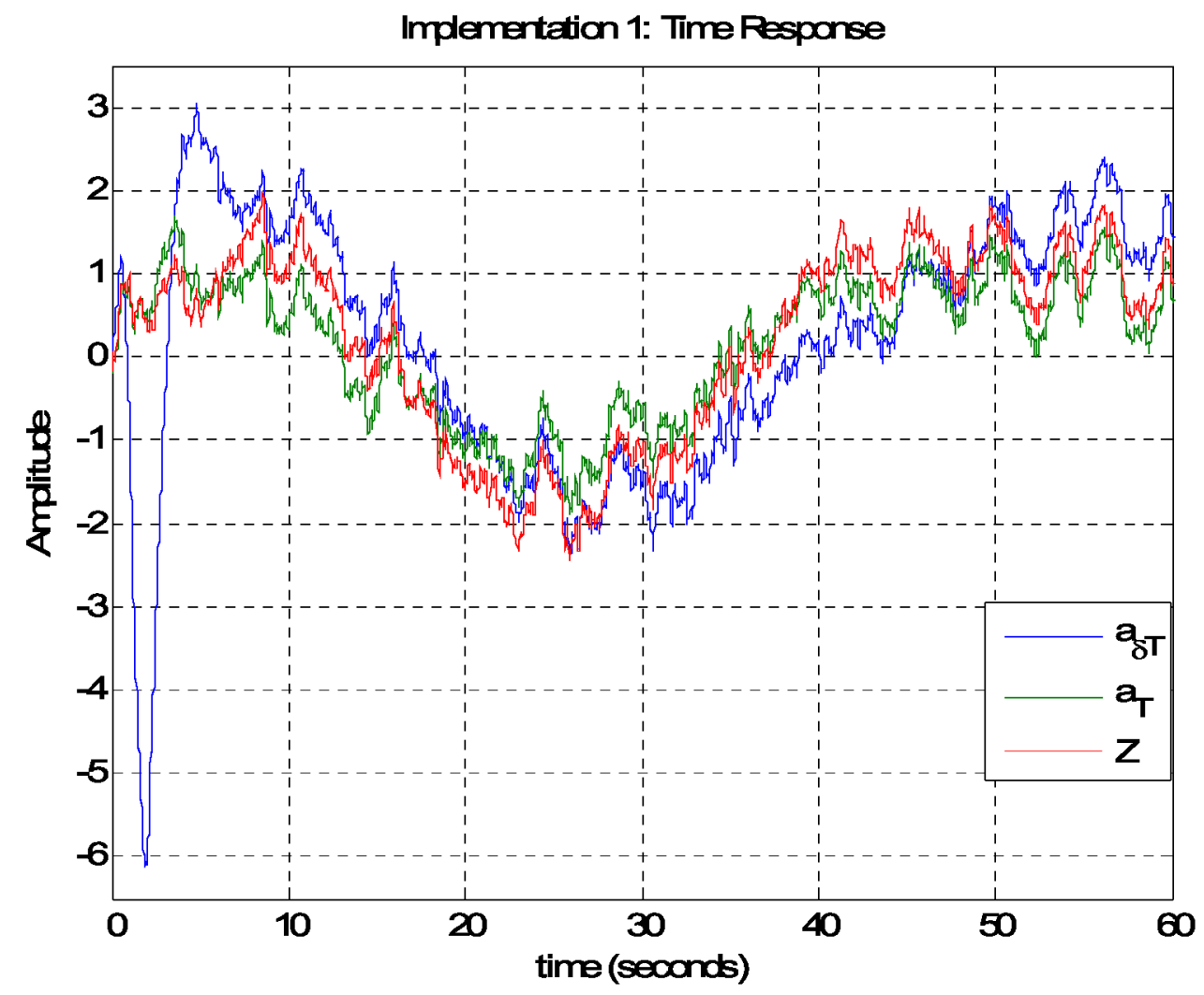

Figure 12: Implementation 1, time response of the aircraft's response to turbulence and pilot input $\left(a_{\delta T}\right)$, turbulence alone $\left(a_{T}\right)$, and the output of the algorithm $(Z)$

The initial peaks in $a_{\delta T}$, between 0 and 4 seconds, are not present in $a_{T}$, but do have an effect on the output of the simulation. The motion cueing algorithm modifies this $a_{\delta T}$ signal, conditioning the aircraft's response to pilot input for the motion system. When it is summed with the output from the turbulence channel, the cue effectively represents both pilot input and atmospheric turbulence. Being that the magnitude of $\mathrm{Z}$ is greater than the physical limits of the motion base; a scaling factor will be implemented within the algorithm. A linear scaling of 0.25 would ensure the motion cue $(\mathrm{Z})$ remains in bounds. 


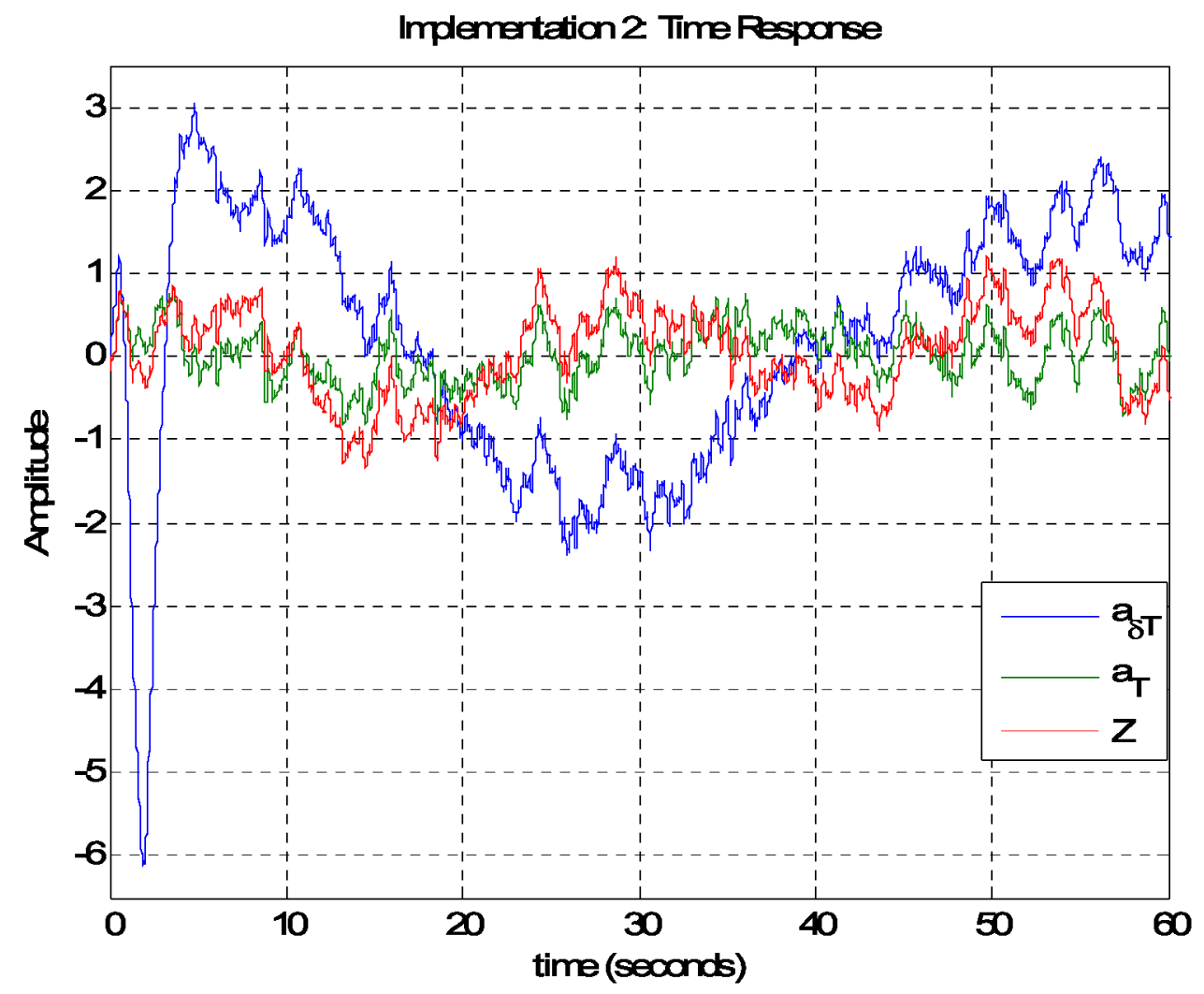

Figure 13: Implementation 2, time response of the aircraft's response to turbulence and pilot input $\left(a_{\delta T}\right)$, turbulence alone $\left(a_{T}\right)$, and the output of the algorithm $(Z)$

When the cueing filter is applied to the turbulence channel, the long period dynamics of the aircraft are washed out from the signal. The turbulence channel has a great deal of impact on the final output of the simulation; therefore $\mathrm{Z}$ no longer retains the long period dynamics. The use of a washout filter would be desirable if the signal being filtered had divergent tendencies. This was not observed when testing the simulation, even over long periods of time.

If it is determined that the pilot's perception of the long period dynamics of the aircraft's turbulence response is unnecessary, then the cueing filter may be applied to the turbulence channel, as in implementation 2 . This motion has a period of about 50 seconds, generating little acceleration when compared to $\mathrm{a}_{\mathrm{T}}$. Since the short period is most prominent in the signal, the pilot of the simulator most likely won't sense the long period motion.

Further evaluation of the two implementations was based on the power spectral density of the motion cues. $a_{T}$ doesn't have as much power over the lower frequencies as both $\mathrm{Z}$ and $\mathrm{a}_{\delta \mathrm{T}}$. Pilot input, in general, is at a much lower frequency than atmospheric turbulence. For implementation 1, the PSD of the motion cue is quite similar to the full order aircraft model, see figure 14. The zoomed portion of Figure 14 shows the PSD remains consistent even in higher frequencies. The plots of $\mathrm{Z}$ and $\mathrm{a}_{\delta \mathrm{T}}$ become virtually indistinguishable. 

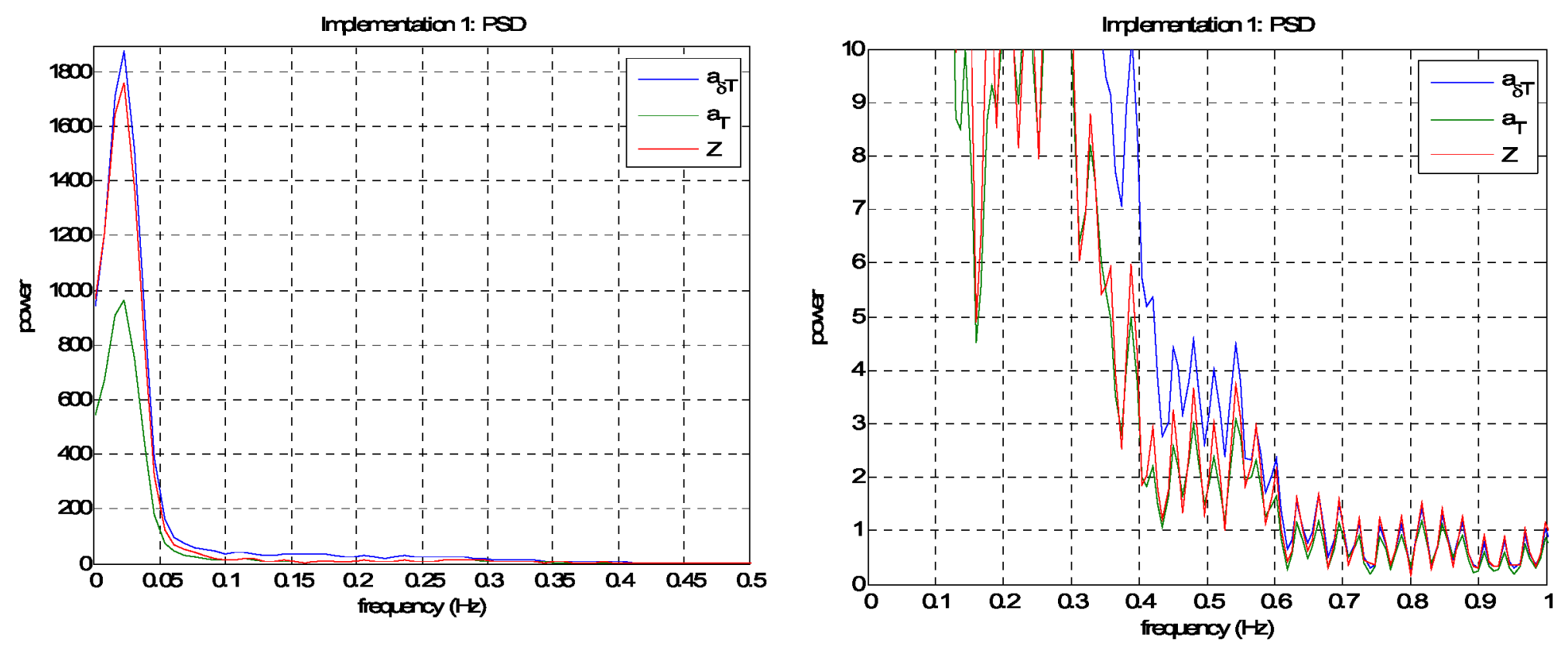

Figure 14: Implementation 1, PSD of the aircraft's response to turbulence and pilot input $\left(a_{\delta \mathrm{T}}\right)$, turbulence alone $\left(a_{T}\right)$, and the output of the algorithm $(Z)$
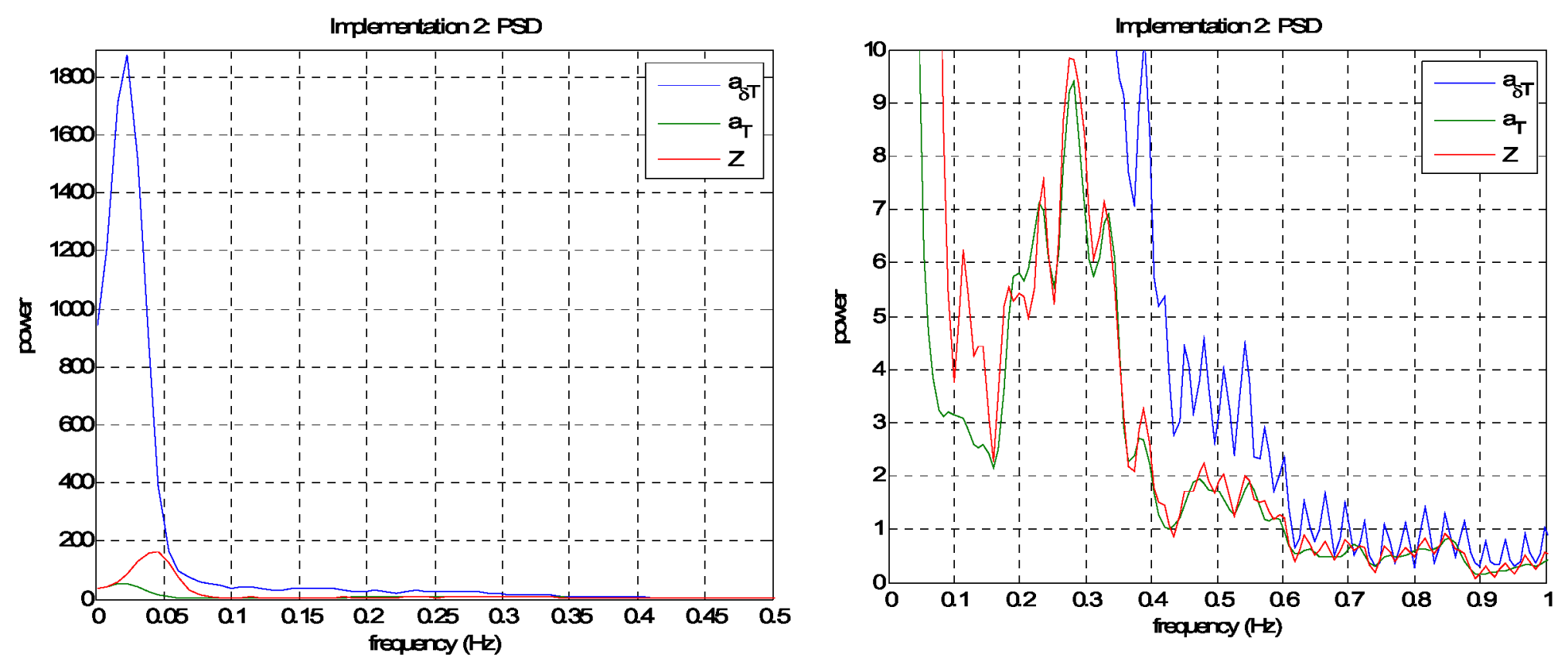

Figure 15: Implementation 2, PSD of the aircraft's response to turbulence and pilot input $\left(\mathrm{a}_{\delta \mathrm{T}}\right)$, turbulence alone $\left(a_{T}\right)$, and the output of the algorithm $(Z)$ 
The PSD plot from implementation 2 shows the full effects of adding the cueing filter to the turbulence channel. The low frequency power of $a_{t}$, after the filter, is reduced a great deal. This then diminishes the low frequency power of the cue. The PSD of the higher frequency cues $(\mathrm{Z})$ do not have the same power as the FOAC output $\left(\mathrm{a}_{8 \mathrm{~T}}\right)$.

\section{Conclusions and Future Research}

The paper has illustrated analytically an approach to providing more appropriate motion cues to pilots of flight simulators while the simulated aircraft is subjected to atmospheric turbulence. This technique could be applied to other high frequency disturbances such as aeroelastic effects. In the very near future a study will be conducted, using a number of pilots on the NASA LaRC VMS, to measure the effects of atmospheric turbulence and its simulation on pilot performance. Subjective evaluations will also be conducted.

\section{References}

Telbam, R. J., and Cardullo, F. M., "Motion Cueing Algorithm Development: Human-Centered Linear and Nonlinear Approaches," NASA CR-2005-213747, 2005

Reid, L. D., and Robinson, P. A., "Augmentation Flight Simulator Motion Response to Turbulence," Journal of Aircraft, Vol. 27, No. 4, 1990

Garrood, S. T., and Reid, L. D., "Pilot Evaluations of Augmented Flight Simulator Motion," Journal of Aircraft, Vol. 31, No. 4, July-Aug. 1994 